\title{
Spectroscopic and physical parameters of Galactic O-type stars
}

\section{Effects of rotation and spectral resolving power in the spectral classification of dwarfs and giants $\star, \star \star$}

\author{
N. Markova ${ }^{1}$, J. Puls ${ }^{2}$, S. Scuderi ${ }^{3}$, S. Simón-Díaz ${ }^{4,5}$, and A. Herrero ${ }^{4,5}$ \\ ${ }^{1}$ Institute of Astronomy with NAO, BAS, PO Box 136, 4700 Smolyan, Bulgaria \\ e-mail: nmarkova@astro.bas.bg \\ 2 Universitäts-Sternwarte, Scheinerstrasse 1, 81679 München, Germany \\ e-mail: uh101aw@usm.uni-muenchen.de \\ 3 INAF - Osservatorio Astrofisico di Catania, via S. Sofia 78, 95123 Catania, Italy \\ e-mail: scuderi@oact.inaf.it \\ 4 Instituto de Astrofísica de Canarias, 38200 La Laguna, Tenerife, Spain \\ e-mail: [ssimon; ahd] @iac.es \\ 5 Departamento de Astrofísica, Universidad de La Laguna, 38205 La Laguna, Tenerife, Spain
}

Received 20 October 2010 / Accepted 17 February 2011

\begin{abstract}
Context. The modern-era spectral classification of O-stars relies on either the Walborn or the Conti-Mathys scheme. Since both of these approaches have been developed using low-quality photographic data, their application to high-quality digital data might not be straightforward and be hampered by problems and complications that have not yet been appreciated.

Aims. We investigate the correspondence between photographic and digital classification of low luminosity O-type stars (dwarfs and giants) of solar metallicity.

Methods. Using high-resolution spectra obtained with the ESO/MPG $2.2 \mathrm{~m}$ telescope in La Silla and following the premises of the Walborn and Conti classification schemes, we determined the spectral types and luminosity classes of 19 Galactic O-type stars and compared them to those attributed by Walborn and Mathys based on low-quality data.

Results. Our analysis reveals that the morphological spectral types assigned using high-resolution data are systematically later (by up to 1.5 subtypes) then those attributed by Walborn. By means of line-profile simulations, we show that part of this discrepancy is more likely caused by the combined effect of stellar rotation and high spectral resolution on the depth of helium lines used as spectral type indicators. In addition, we demonstrate that at least for narrow-lined stars the "rotational effect" does not disappear when the high-resolution spectra are degraded to the resolution of the Walborn standards. We also find evidence of a systematic difference between our high-resolution quantitative spectral types and those assigned by Mathys.

Conclusions. Rotation and spectral resolution are important third parameters in the spectral classification of O-type stars. To obtain reliable spectral classes within the Walborn approach, the unknown and the standard spectra must be compared at the same resolution and $v \sin i$. Owing to resolution effects, the Conti approach might also need to be updated.
\end{abstract}

Key words. stars: early type - stars: fundamental parameters - binaries: spectroscopic

\section{Introduction}

Despite their scarcity, massive stars play an important role in the history of the Universe. They are the main engines driving the chemical and dynamical evolution of galaxies, enriching the interstellar medium with heavy elements, creating $\mathrm{H}_{\text {II }}$ regions, and exploding as supernovae. In the distant Universe, they dominate the integrated UV radiation in young galaxies. Massive stars are possibly key objects for studying and understanding exciting phenomena such as the cosmic reionisation and $\gamma$-ray bursters.

In the past decade, our knowledge of the physics of massive stars has drastically improved because of important developments in massive star modelling (both in their interiors

^ Based on observations collected at the European Organisation for Astronomical Research in the Southern Hemisphere, Chile, under programme ID 072.D-0196.

$\star \star$ Appendices $\mathrm{A}$ and $\mathrm{B}$ are only available in electronic form at http://www. aanda.org and outer envelopes), and continuously higher quality observations collected from the ground and space. Despite the tremendous progress made so far, a number of new challenging issues (regarding both theory and observations) have emerged that need to be addressed urgently. Among these are the "weak wind problem" in O-type stars, the wind momenta of Galactic B-supergiants being significantly lower than predicted, the behaviour of mass loss at the bi-stability jump, the effects of rotation and magnetic fields, and wind clumping. For a comprehensive review of the status-quo of massive star research, with emphasis on radiatively driven mass loss, the interested reader is referred to Puls et al. (2008).

For the particular case of O-stars in our Galaxy, the investigation of the aforementioned open problems, especially those related to stellar winds, requires a large sample of objects to be analysed to diminish the error caused by the uncertain distances. Since Galactic O-type stars constitute an important reference frame to investigate metallicity effects, this point is also crucial 
for extragalactic surveys. Though easy accessible with small and medium size telescopes, Galactic O-stars have not been widely observed to derive consisitent stellar and wind parameters from quantitative spectroscopy, and no more than 50 objects have been studied in detail (Herrero et al. 2002; Repolust et al. 2004; Bouret et al. 2005; Martins et al. 2005b; Dufton et al. 2006; Marcolino et al. 2009).

The main goal of our project is to increase the number of Galactic O-type stars with reliably determined physical parameters, using high-quality spectra and applying the methods of quantitative spectroscopic analyses. On the basis of new data and incorporating similar data from previous investigations, we plan to reinvestigate the latest calibrations for these stars (Martins et al. 2005a), to address the important question of macroturbulence and its effect on the derived $v \sin i$, and to investigate the weak wind problem in stars of solar metallicity. Since the absolute calibration of the various relationships between physical parameters and observed spectral characteristics (e.g., line strengths or, equivalently, spectral types) requires reliable estimates of all involved quantities, a thorough investigation of the accuracy of the spectral types and luminosity classes initially assigned to our targets and an update of their binary/multiplicity status are required.

In this first paper of the series, the main results from the spectral classification of the low luminosity stars in our sample (dwarfs and giants) are presented. A second paper providing the physical properties of these stars derived by means of the latest version (V10.1) of the state-of-the-art model atmosphere code FASTWIND (Puls et al. 2005) is currently in preparation and will be published soon (Markova et al. 2011, hereafter Paper II). A third paper concentrating on the spectral classification and model atmosphere analysis of the supergiants in our sample is planned for the near future.

The present paper is structured as follows. In Sect. 2, we describe the stellar sample and the observational material underlying the project. In Sect. 3.1, we outline the main steps in our classification procedure. Section 3.2 deals with the possible binarity/multiplicity among the sample stars. In Sect. 4, the correspondence between our high-resolution morphological and quantitative classification and the classifications attributed by Walborn $(1972,1973)$ and Mathys (1988) for stars in common is investigated, and a possible explanation of the derived discrepancies is provided. The spectral types determined in the framework of the Walborn and Conti-Mathys schemes from our high-resolution observations are compared in Sect. 5. Finally, in Sect. 6, we summarise the main results of our study and comment on some implications these findings might have in the future. Extensive comments on each sample star and an atlas of the corresponding high resolution spectra are presented in the appendix.

\section{Observations and data reduction}

The total sample consists of 40 bright ( $V \leq 10 \mathrm{mag}$ ) O-type stars in the Milky Way originally classified as dwarfs, giants, and supergiants, with spectral types ranging from $\mathrm{O} 3$ to B0.5. The targets were observed with the FEROS spectrograph (Kaufer et al. 1999) at the ESO/MPG $2.2 \mathrm{~m}$ telescope in La Silla on the 4th and 5th of February, 2004. Each spectrum covers a wavelength range from about 350 to about $920 \mathrm{~nm}$ with a spectral resolution $R=48000$. Exposure times ranging from 400 to $2500 \mathrm{~s}$ were used to obtain a typical signal-to-noise ratio (hereafter $S / N$ ) of 150-200 per resolution element. One-dimensional, wavelengthcalibrated spectra were extracted using the FEROS pipeline.
For our present study, we considered only the subset of the sample dwarfs and giants (19 in total) to diminish the effects of stronger winds on the outcome of the classification analysis. The stellar IDs along with our present and previous spectral classifications by Walborn (Walborn 1972, 1973; Walborn et al. 2002) and Mathys (1988) are presented in Table 1. We note that for three sample stars that have been classified by neither Walborn nor Mathys, previous classifications based on lowresolution photographic spectra and MK standards were adopted (Col. 4 of Table 1).

\section{General comments}

\subsection{Spectral classification}

The modern-era O-star spectral classification relies on either the Walborn or the Conti-Mathys schemes, both being somewhat related to the MK system defined essentially in terms of standard stars. In particular, based on a careful investigation of MK standards observed at a spectral resolution twice that of the MK atlas, Walborn (1971, 1972, 1973) developed a two-dimensional empirical system for O-type stars of solar metallicity. This system was additionally worked out by Walborn and coworkers, and transferred to digital data of similar (Walborn \& Fitzpatrick 1990) or higher (Walborn et al. 2002) resolution. Since the Walborn approach relies on a visual inspection of the spectra, and the visibility of certain lines and certain line ratios are used as criteria, it is usually referred to as a morphological classification. Independently of and simultaneously with the morphological approach, Conti and collaborators (Conti \& Alschuler 1971; Conti \& Leep 1974; Conti \& Frost 1977) developed an alternative method based on logarithmic ratios of equivalent widths $(E W)$ of certain helium and metal lines, calibrated against MK spectral types. The quantitative classification system of Conti was refined by Mathys (1988, 1989).

To classify our targets, we applied both the Walborn and the Conti-Mathys systems. In the former case, we considered the visual impression of our high-resolution spectra and, following the premises of Walborn \& Fitzpatrick (1990), performed a morphological classification. However, since the line visibility depends directly on the data quality, we refrained from using criteria related to the initial appearance of certain lines and concentrated only on eye-estimated line ratios, to avoid possible systematic effects caused by the higher resolution (and $S / N$ ) of our spectra. We primarily used the He I to He II line ratios (particularly those of both He I+II $\lambda 4026$ to He II $\lambda 4200$ and He I $\lambda 4471$ to He II $\lambda 4541$ ) to determine the spectral subtype, except for the hottest stars where criteria based on the nitrogen ionization equilibrium (Walborn et al. 2002) were instead applied. For the luminosity class, we followed the Walborn approach and used different criteria at different subtypes. For example, at earlier types we considered the effect of luminosity on the strength of both the N IV $\lambda 4058$ and He II $\lambda 4686$ emission, and the N V $\lambda \lambda 4604,4620$ absorption. At spectral types from $\mathrm{O} 6$ to O8, the main luminosity indicators are He II $\lambda 4686$ and N III $\lambda \lambda 4634-4640-4642$ : in dwarfs, the former line appears strongly in absorption, accompanied by weak N III emission - the V((f)) category; in giants, the He II $\lambda 4686$ absorption weakens and can even vanish while the $\mathrm{N}$ III $\lambda \lambda 4634$ 4640-4642 emission strengthens - the III(f) category. At types O9-B0, the primary luminosity criterion is the strength of the $\mathrm{Si}$ IV doublet around $\mathrm{H}_{\delta}$, whose absorption increases with luminosity. 
Table 1. Sample stars along with spectral classification.

\begin{tabular}{|c|c|c|c|c|c|c|c|c|c|}
\hline \multirow[t]{2}{*}{ Star } & \multicolumn{4}{|l|}{ Previous } & \multirow[t]{2}{*}{$\bar{c} v \sin i$} & \multicolumn{3}{|l|}{ Present study } & \multirow[b]{2}{*}{ Spect. $^{b}$} \\
\hline & Walborn & Mathys & GHS & Intfr. $^{a}{ }$ Spect. $^{d}$ & & Morph. $\left(R_{48000}\right)$ & Quant. & Morph. $\left(R_{4000}\right)$ & \\
\hline HD 64568 & O3 V((f*)) & & & VS & $100, \mathrm{M}$ & O3 V $\left(\left(f^{*}\right)\right)$ & & O3 V((f*)) & SS \\
\hline HD 93204 & O5 V((f)) & O5.5 V((f)) & & VS & $115, \mathrm{M}$ & O6 V((f)) & $05.5 \mathrm{~V}$ & O6 V((f)) & SS \\
\hline HD 93843 & O5 III(f) & O5.5 III(f) & & VS & $100, \mathrm{P}$ & O6 III(fc) & O5.5 III & O5.5 III(fc) & SS \\
\hline CPD -592600 & O6 V((f)) & & & VS & $142, \mathrm{H}$ & O6 V((f)) & O6.5 V/III & O6 V((f)) & SS \\
\hline HD 63005 & & & O6 V((f)) & & $74, \mathrm{H}$ & $07.5 \mathrm{~V}((\mathrm{f}))$ & O6.5 V & $\mathrm{O} 7 \mathrm{~V}((\mathrm{f}))$ & SS \\
\hline HD 152723 & O6.5 III(f) & O7 III(f) & & $\mathrm{VD}, \mathrm{SB} 1 ?$ & $123, \mathrm{P}$ & O6.5 III(f) & O6.5 III & O6.5 III(f) & SB1 \\
\hline HD 93160 & O6 III(f) & & & VS, SB1? & 205, U & O7 III(f) & $\mathrm{O} 7 \mathrm{~V}$ & O7 III(f) & SB1 \\
\hline HD 94963 & O6.5 III(f) & & & VS & $90, \mathrm{CE}$ & O7.5 II(f) & O7-7.5 I/III & O7.5 III(f) & SS \\
\hline CPD - 58 2620 & O6.5 V((f)) & & & VS & $60, \mathrm{M}$ & O8 V((f)) & O7 III & $07.5 \mathrm{~V}((\mathrm{f}))$ & SS \\
\hline HD 69464 & O6.5 Ib(f) & & & VS & $82, \mathrm{P}$ & O7.5 II(f) & O7-7.5 III & O7 III(f) & SB2? \\
\hline HD 93222 & O7 III(f) & & & VS & $77, \mathrm{P}$ & O8 III (f) & O7 III & O7.5 III(f) & SS \\
\hline HD 91824 & O7 V((f)) & O7 V ((f)) & & VS & $65, \mathrm{H}$ & O8 V ((f)) & $06.5 \mathrm{~V}$ & O7 V ((f)) & SS \\
\hline CD -434690 & & O7.5 III(f) & & & $120, \mathrm{M}$ & O7 III(f) & O6.5 V/III & O7 III(f) & SS \\
\hline HD 92504 & $08.5 \mathrm{~V}((\mathrm{n}))$ & $09 \mathrm{~V}$ & & & $200, \mathrm{U}$ & $08.5 \mathrm{~V}$ & O9 III/V & $08.5 \mathrm{~V}$ & SS \\
\hline HD 151003 & O9 II & O9.5 III & & VS, SB1? & $120, \mathrm{U}$ & O9 II & O9 III & O9 II & SB1 \\
\hline HD 152247 & O9.5 II/III & O9.5 III & & VS, SB2 & $120, \mathrm{P}$ & O9 II & O9 III & O9 II & SB2 \\
\hline HD 302505 & & & O8.5 III & & $80, \mathrm{M}$ & O9 III & O8 III & O9 III & SS \\
\hline CPD - 444865 & & O9.7 III & & & $80, \mathrm{M}$ & B0 III & O9.5 III & B0 III & SS \\
\hline HD 69106 & & & B0.5 IVnn & & $325, \mathrm{H}$ & B $0.2 \mathrm{~V}$ & O9.7 & B $0.2 \mathrm{~V}$ & SS \\
\hline
\end{tabular}

Notes. Low-resolution quantitative and morphological classification from Mathys (1988) and from Walborn (Walborn 1972, 1973; Walborn et al. 2002) and Garrison et al. (1977, GHS), respectively; high-resolution quantitative classification from the present study together with morphological classification based on the original data $(R=48000)$ and degraded ones $(R=4000)$. Additional information about $v \sin i$ (in km s $\mathrm{s}^{-1}$; macroturbulence not accounted for) and binarity status of the stars is also provided. Binarity status from speckle interferometry (Mason et al. 2009) and spectroscopy (previous and present). ${ }^{(a)}$ Results from the speckle interferometric survey of Mason et al. (2009): VD = visually double; VS = visually single object. ${ }^{(b)}$ Spectroscopic binarity status: SS = spectroscopically single object; SB1/SB2 = single-/double-lined spectroscopic binary; SB1/2? = possible spectroscopic binary.

References. for $v \sin i$ - estimates: $\mathrm{H}=$ Howarth et al. (1997); M = Markova et al. 2011 (Paper II); P = Penny (1996); U = Uesugi \& Fukuda (1995); CE $=$ Conti \& Ebbets (1977). See text for further information.

Our quantitative classification has been performed by exploiting the logarithmic ratios $\log W^{\prime}=\log E W(\mathrm{HeI} \lambda 4471)$ - $\log E W($ HeII $\lambda 4542)$, which unambiguously determines the spectral type, and $\log W^{\prime \prime}=\log E W(\operatorname{SiIV} \lambda 4089)-$ $\log E W(\mathrm{HeI} \lambda 4143)$, which is the main luminosity indicator for stars of spectral types 07 and later (Conti \& Alschuler 1971). For stars earlier than $\mathrm{O} 7$, on the other hand, the negative luminosity effect in He II $\lambda 4686$ with a demarcation line between class $\mathrm{V}$ and III set at $\log E W\left(\right.$ HeII4686) $=-0.25^{1}$ was used (Mathys 1988 and references therein). The equivalent widths of the lines involved in the quantitative classification are provided in the appendix (Table A.1). The accuracy of these estimates ranges from 0.02 to $0.07 \mathrm{dex}$, with lower values being typical of stronger lines. The errors in $\log W^{\prime}$ and $\log W^{\prime \prime}$ are between 0.05 and 0.07 dex (i.e., less than half a subtype), and about 0.1 dex, respectively.

Our final morphological and quantitative classifications are listed in Cols. 7 and 8 of Table 1. Column 6 gives $v \sin i$ estimates as adopted in the present study, primarily drawn from Penny (1996) and Howarth et al. (1997) ${ }^{2}$, if present. Otherwise, individual data provided by Uesugi \& Fukuda (1995) or derived in Paper II were used instead.

\subsection{Spectroscopic binarity}

It is well known that spectroscopic binarity can significantly bias the classification of stars, modifying the visual appearance of the

\footnotetext{
$1 \log E W($ HeII4686) larger than -0.25 determines the luminosity class V.

2 With respect to our targets, the consistency between the two datasets is good, to within $\pm 10 \mathrm{~km} \mathrm{~s}^{-1}$.
}

spectrum and altering the observed equivalent widths of lines used as spectral type and luminosity class diagnostics. This point is particularly important in the case of hot massive stars where the number of double/multiple systems seems to be very large (e.g., Mason et al. 2009, and references therein).

To investigate the binary status of our targets, we checked the literature for known/suspected binaries among our sample stars and found that three of them - HD 93160, HD 151003 (Gies 1987), and HD 152723 (Fullerton et al. 1996) - have been suspected to be single-lined spectroscopic binaries (SB1), because of their $V_{\mathrm{r}}$-variability. Large variations in $V_{\mathrm{r}}$ were also detected for another star, HD 64568 (Solivella \& Niemela 1986). Since $V_{\mathrm{r}}$ - variability can be caused by binary motion, but may also originate from stellar pulsations and/or wind effects, we decided to keep the $V_{\mathrm{r}}$-variables in our target list for further clarification. Meanwhile, we rechecked the literature for newer results and found that one of our sample stars (HD 152247) had been recognized as a double-lined spectroscopic binary (Sana et a. 2008); another one (HD 152723) was resolved as a close double system via $V$-band speckle interferometry, while for other 12 objects (flagged with "VS" in Col. 5 of Table 1) a "null companion detection" in the angular separation range 0. " $035 \leq \rho \leq 1$." 5 and $\Delta m \leq 3 \mathrm{mag}$ was reported (Mason et al. 2009 and references therein). We kept the two binaries in the present sample with the primary goal of investigating the effect of binarity on the outcome of our spectral classification.

We also tried to constrain the spectroscopic binarity status of all sample stars by our own. To this end, we adopted the following criteria as indications for possible binarity: (i) composite spectrum; (ii) discrepant shifts, widths and/or strengths of lines 


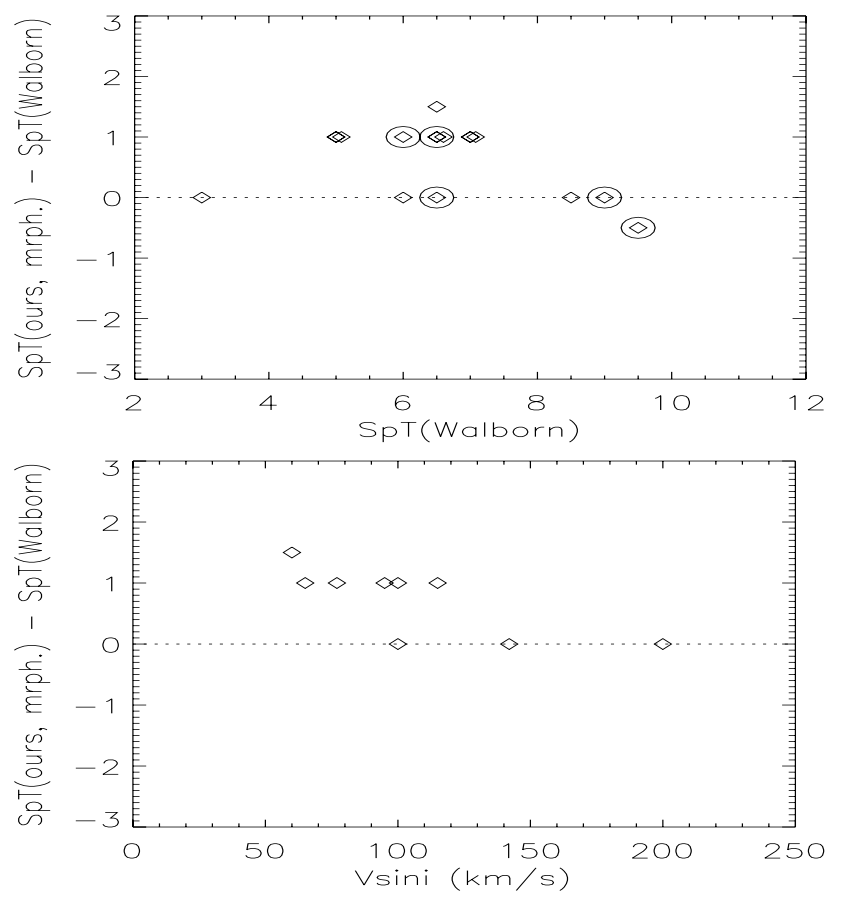

Fig. 1. Top: comparison of our high-resolution morphological spectral types and those attributed by Walborn by means of low-resolution photographic spectra $(\Delta \lambda=1.2 \AA)$, for 14 stars (dwarfs and giants) in common. The X-scale corresponds to spectral types from $\mathrm{O} 2$ to B2. Stars considered/suspected to be SBs are additionally marked with large circles. Bottom: the spectral type discrepancy as a function of $v \sin i$. To eliminate binarity effects on the observed $v \sin i$, stars considered/suspected to be SBs have been discarded. Note that the adopted $v \sin i$-estimates do not account for the effects of macroturbulence.

in the spectrum; (iii) large differences in $V_{\mathrm{r}}$ and $v \sin i$ when measured at different observational epochs; (iv) periodic variations in photometry and/or radial velocity; and (v) discrepant spectral classification from various observational epochs. Since physical phenomena that differ from binarity can give rise to periodic photometric and $V_{\mathrm{r}}$-variability while discrepant spectral classifications might be due to third parameter effects (see below), these last two criteria were considered only as suggestive.

A comprehensive description of our findings for each star is presented in the appendix. The spectroscopic status of the targets is listed in Col. 10 of Table 1: "SS" denote spectroscopically single objects, "SB2" are double-lined, and "SB1" single-lined spectroscopic binaries.

In summary, following our approach we confirm the binary status of HD 152723 and HD 152247, provide strong evidence of a companion in HD 93160 and HD 151003, and suspect duplicity in HD 69464.

\section{Comparison with previous classifications from low resolution spectroscopy}

\subsection{The present high-resolution morphological classification and the Walborn results}

To investigate whether the results derived by means of the Walborn scheme might be influenced by the spectral resolution, we compared our morphological spectral types with those attributed by Walborn, based on low-resolution $(\Delta \lambda=1.2 \AA)$ photographic spectra, for 14 stars in common (see Col. 2 of Table 1). As illustrated in Fig. 1 (top panel), the high-resolution digital classification tends to result in somewhat later (by up to 1.5 subtypes) spectral types than those derived by Walborn. This discrepancy is larger than the error in the morphological classification (typically less than one subtype), thus significant. An interpretation in terms of variable wind effects or possible binarity does not seem likely, since all targets are low luminosity objects, i.e., have low-density winds, and the majority of them do not appear to have a companion. In addition, the distribution of the data suggests that the derived discrepancy might be present at intermediate subtypes only. Since the main spectral type indicator at these subtypes is the eye-estimated ratio of either He I+II $\lambda 4026$ to $\mathrm{He}$ II $\lambda 4200$, or He I $\lambda 4471$ to He II $\lambda 4541$, this result implies in turn that within the high-resolution digital spectra either He I+II $\lambda 4026$ and He I $\lambda 4471$ appear to be visually stronger or He II $\lambda 4200$ and He II $\lambda 4541$ appear to be visually weaker (or both), compared to their corresponding strengths as estimated from the photographic spectra.

Apart from stars with discrepant spectral types, our analysis shows that there are also others with identical morphological classifications as derived from high- (digital) and low-resolution (photographic) spectra. One of these (HD 64568) is a very early O3 dwarf (see below); two other stars (HD 152723, O6.5 and HD 151003, O9) are considered to be spectroscopic binaries (present study); and the final two are among the fastest rotators in our sample (CPD -59 2600, O6, $v \sin i=142 \mathrm{~km} \mathrm{~s}^{-1}$ and HD 92504, O8.5, $\left.v \sin i=200 \mathrm{~km} \mathrm{~s}^{-1}\right)$. The latter finding is quite intriguing since it might imply that the encountered discrepancy is also related to rotation. We test this possibility in Fig. 1 (lower panel), where the differences in spectral type are plotted against $v \sin i$. At least for our subsample, the spectral type discrepancy indeed refers to stars with relatively slow rotation $\left(v \sin i \leq 120 \mathrm{~km} \mathrm{~s}^{-1}\right)^{3}$. The only exception is HD 64568 with $v \sin i=100 \mathrm{~km} \mathrm{~s}^{-1}$, which, however, has not been classified in terms of the eye-estimated ratios of $\mathrm{He}$ I to $\mathrm{He}$ II but instead exploits the relative strengths of nitrogen lines of different ionisation.

Summarizing, our analysis shows that using high resolution digital spectra of O-type stars of solar metallicity results in somewhat later morphological subtypes, where this discrepancy is more pronounced in narrow-lined stars.

High-resolution against low-resolution morphological luminosity classes. A comparison of the luminosity classes determined in this work with those attributed by Walborn for 14 stars in common shows perfect agreement for all but two stars HD 94963 and HD 69464. This finding suggests that at least for dwarfs and giants the luminosity class criteria within the Walborn scheme are not affected by resolution, rotation, and/or origin of data.

\subsubsection{Influence of rotation and spectral resolution}

That rotation should influence the depths of $\mathrm{He}$ I and He II lines differently is to be expected from the different intrinsic widths of these lines, caused by the Stark effect (linear in He II and quadratic in He I). This point is illustrated in Fig. 2, where synthetic profiles for He I $\lambda 4471$ and He II $\lambda 4541$, as calculated from a FASTWIND model at $T_{\text {eff }}=38 \mathrm{kK}, \log g=3.7$, and $\log Q=-7.72^{4}$, have been convolved with four values of $v \sin i$,

\footnotetext{
${ }^{3}$ Similar results apply for the three stars classified by Garrison et al. (1977), see Table 1, Col. 4.

${ }^{4} Q=\dot{M} / R_{\star}{ }^{1.5}$ is the optical depth invariant for recombination-based diagnostics such as $\mathrm{H}_{\alpha}$, introduced by Puls et al. (1996) to characterise
} 

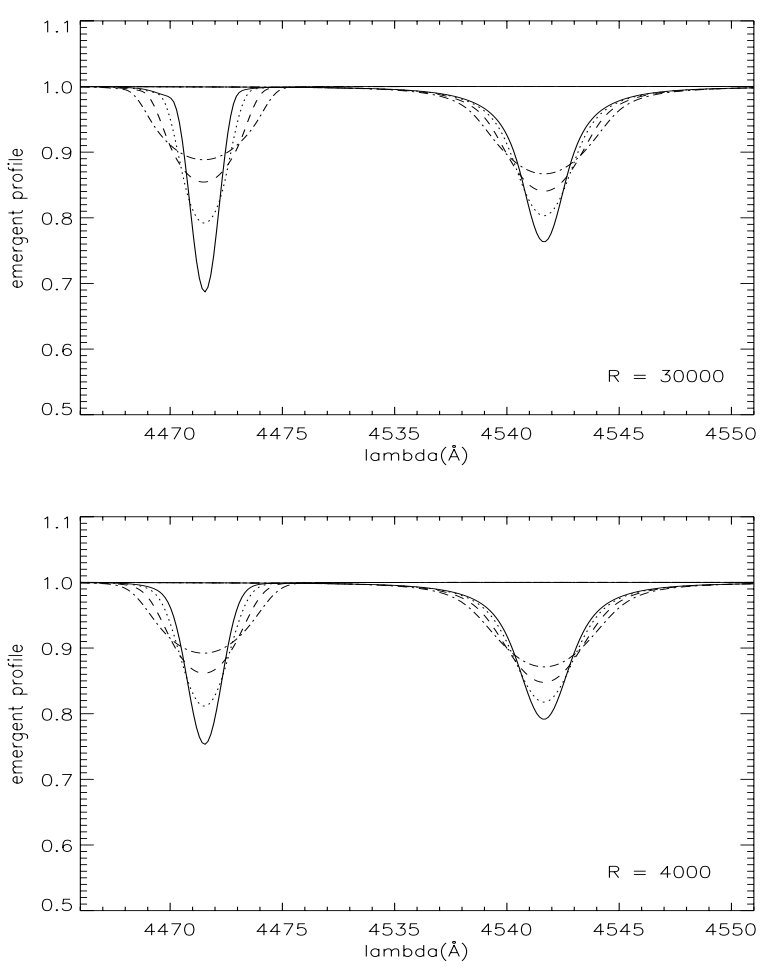

Fig. 2. Examples illustrating the sensitivity of the central depth of $\mathrm{He}$ I $\lambda 4471$ and He II $\lambda 4541$ to stellar rotation $(v \sin i=60$ (solid), 100 (dotted), 150 (dashed), 200 (dash-dotted) $\mathrm{km} \mathrm{s}^{-1}$ ), and spectral resolution $(R=30000$ (top), 4000 (bottom)). Model profiles from FASTwIND, calculated at $T_{\text {eff }}=38 \mathrm{kK}, \log g=3.7$ and $\log Q=-7.72$, with $\log W^{\prime}=-0.19$ corresponding to subtype O6.5.

while keeping the spectral resolution fixed at $R=30000$ (top panel) and 4000 (bottom panel). The main result of our calculations is that at the same temperature, surface gravity, chemical abundances, mass loss rate, and spectral resolving power the eye-estimated ratio of $\mathrm{He}$ I $\lambda 4471$ to $\mathrm{He}$ II $\lambda 4200$ can indicate different spectral types, depending on $v \sin i$. A comparison of the profiles shown in Fig. 2 indicates that the morphological spectral types for fast $\left(v \sin i \geq 150 \mathrm{~km} \mathrm{~s}^{-1}\right)$ and slowly $\left(v \sin i \leq 100 \mathrm{~km} \mathrm{~s}^{-1}\right.$ ) rotating stars can differ by about half (at $R=4000$ ) to about one and a half (at $R=30000$ ) subtypes, where slowly rotating stars display a later subtype. Similar results have been obtained for He I+II $\lambda 4026$ and He II $\lambda 4200$ determining the morphological spectral types from $\mathrm{O} 5$ to O6.5.

Illustrative examples of the combined effect of rotation and high spectral resolution on the visual strength of strategic He I and He II lines are shown in Fig. 3, where the FEROS spectra of three narrow-lined sample stars, originally classified as O6.5-O7 by Walborn, are displayed along with log $W^{\prime}$-values from our high-resolution $E W$ measurements. The central depths of He I $\lambda 4471$ and He II $\lambda 4541$ lines obviously react in the way predicted by our model calculations, and indicate a somewhat later spectral type, namely $\mathrm{O} 8$ to 8.5 depending on $v \sin i$.

Thus, we conclude that the established discrepancy between high- and low-resolution morphological spectral types (mostly for stars of intermediate subtypes) is likely related (at least in part, see below) to the interplay between stellar rotation and spectral resolution leading to different depths of He I and

the strength of the wind. $\log Q=-7.72\left(\dot{M}\right.$ in $M_{\odot} / \mathrm{yr}, R_{\star}$ in $\left.R_{\odot}\right)$ represents the case of a low density wind, originating in a low luminosity O-type star.
He II lines, where the profiles become deeper at lower rotational speeds and higher resolution. We emphasize that for stars with identical intrinsic parameters but different rotational speeds, there will be a decisive difference in spectral types derived by either morphological or quantitative classification: whereas the morphological types will vary according to rotational (and resolution) effects, types derived by a quantitative classification will be rather unique, since rotational broadening preserves the $E W$ and thus $W^{\prime}$.

\subsubsection{The modern-era morphological classification}

It is well known that the overall appearance of a stellar spectrum is strongly dependent on its resolving power. Because of this, the modern-era morphological classification is usually performed using (original) spectra that have been degraded to the resolution of the standards. In this process, one expects the morphology of the degraded spectra to closely resemble that of the low resolution classification standards. To our knowledge, however, this expectation has not been so far checked using real data.

For a first test of this issue, the original, high-resolution spectra of our targets were degraded to $R=4000^{5}$ (using the IDL procedure "rebin.pro"), and subsequently classified following the Walborn criteria. In Fig. 4, we confront the spectral types obtained in this way to those attributed by Walborn for stars in common. Although the agreement between the two data sets has improved (compared to the one shown in the top panel of Fig. 1), the spectral types based on the degraded spectra (Col. 9 of Table 1) still tend to be somewhat later than those derived by means of the photographic classification, where again this discrepancy is more significant in narrow-lined stars. To check whether this finding depends on the procedure used to degrade the spectra, we repeated the process using an own IDL procedure based on convolution by Fourier-transforms, and obtained similar results.

The effect of significantly different spectral resolving power on the overall appearance of the spectrum is illustrated in Fig. 5, where the original, high-resolution spectrum of one sample star with relatively narrow lines $\left(\mathrm{CPD}-582620, v \sin i=60 \mathrm{~km} \mathrm{~s}^{-1}\right)$, classified as O6.5 by Walborn (1973), is shown together with its rebinned spectrum ${ }^{6}$. Even at this low resolution, the spectrum of CPD -582620 obviously does not resemble that of HD 93146, the classification standard of $06.5 \mathrm{~V}$, but is similar to that of 15 Mon, the classification standard for O7. Thus, and at least for the case of narrow-lined stars of intermediate spectral type, it appears that degrading the high-resolution spectra to the resolution of the Walborn standards does not necessarily guarantee that they will resemble the morphology of the corresponding standards, since in this case the comparison objects (the one under consideration and the standard) might have different $v \sin i$. The larger the difference, the larger the offset in derived spectral type.

The results outlined above are somewhat surprising since (i) we degraded our spectra to the resolution of the photographic data, and (ii) we classified the same stars as Walborn, i.e., the resolution and rotational effects should be the same in both data

\footnotetext{
5 which matches the quality of the photographic spectra used by Walborn, namely $\Delta \lambda=1.2 \AA$.

${ }^{6}$ Here the original spectrum has been degraded to $R=3000(\Delta \lambda=$ $1.5 \AA$ ) to match the quality of the standards shown in the Walborn and Fitzpatrick atlas.
} 


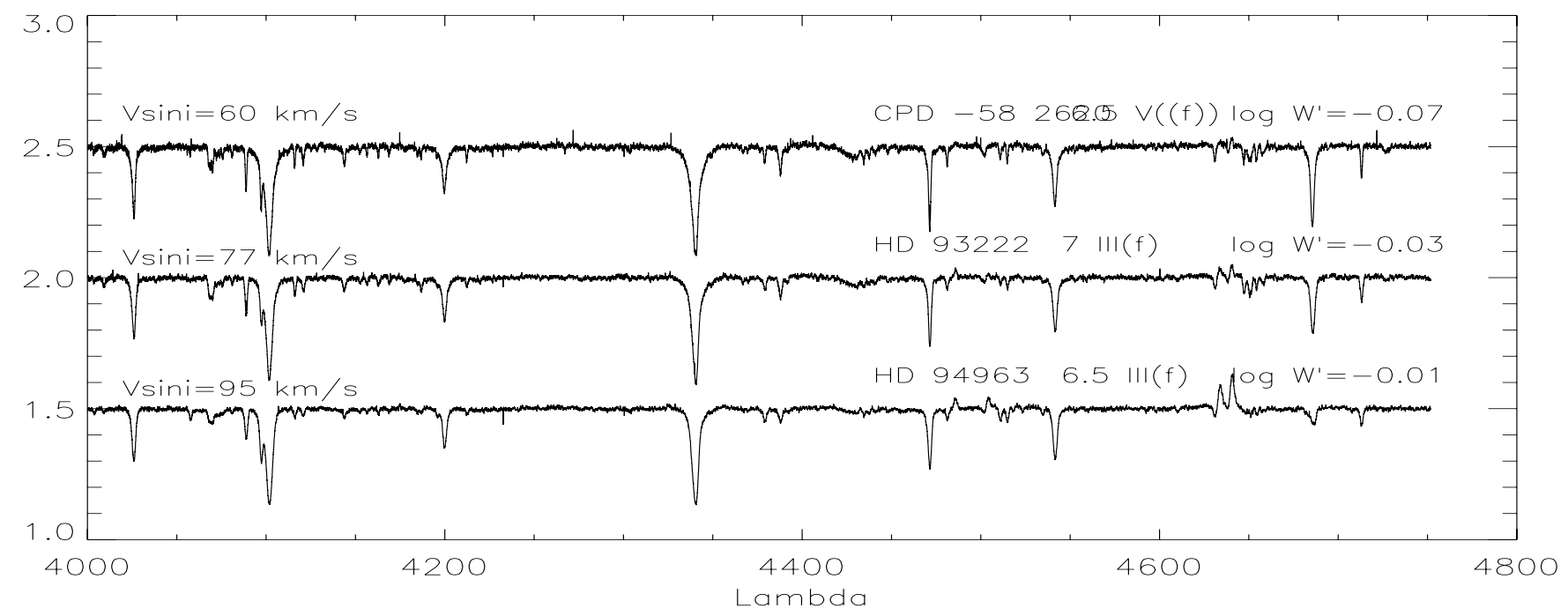

Fig. 3. A set of FEROS spectra illustrating the combined effect of rotation and high spectral resolution on the central depth of the strategic He I $\lambda 4026,4471$ and He II $\lambda 4200,4541$ lines, for stars of similar spectral type (as attributed by Walborn) and similar log $W^{\prime}$, as measured from our high-resolution data. Note that in the Walborn scheme the O7 subtype is defined by He I $\lambda 4471 \approx \mathrm{He}$ II $\lambda 4541$, whereas in our spectra $\mathrm{He}$ I $\lambda 4471$ is always deeper than He II $\lambda 4541$ (although its $E W$ is actually smaller, $\log W^{\prime} \leq 0$ ), indicating a spectral type of O8-8.5.

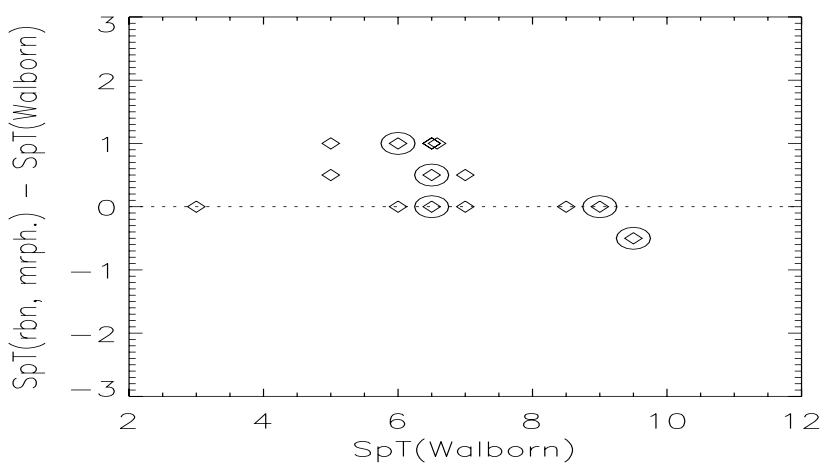

Fig. 4. Comparison of our morphological spectral types, based on the high-resolution spectra degraded to $R=4000$, and those attributed by Walborn using low-resolution photographic spectra $(\Delta \lambda=1.2 \mathrm{~A})$ for 14 stars in common. The X-scale corresponds to spectral types from $\mathrm{O} 2$ to B2. Stars considered/suspected to be SBs are marked with large circles.

sets $^{7}$. Since there are still differences, additional effects must be at work. What might these effects be?

i) The remaining discrepancies shown in Fig. 4 might be caused by the intrinsic differences in the classification techniques applied in the photographic and digital classifications: while in the former case the eye responds to the total flux transmitted/blocked by the investigated lines (recalling, that in the early seventies of the last century the classification was performed by viewing the spectrographs through a microscope), in the latter it is guided by the central depths of the lines. Consequently, both estimates might deviate from each other to some degree, particularly at intermediate subtypes where the differences in line strength are smaller than in other regions.

\footnotetext{
7 Note that the corresponding spectral types might still be biased against the standards due to rotational effects, but both (ours and Walborn's) in the same way, see below.
}

ii) Since O-stars are often embedded in emitting gas, underestimated nebula contamination of He I lines in the photographic spectra might lead to systematically different classifications from those originating from the high-resolution digital data.

iii) Finally, there is a certain chance of line profile variability. Owing to the systematic character of the discrepancy, we consider this possibility, however, less likely.

Thus, we have to conclude that part of the discrepancies between morphological classifications attributed using high-resolution digital and low-resolution photographic spectra (Fig. 1) is due to some additional effects to resolution and rotation alone. This point was also discussed by Walborn et al. (2010).

\subsection{The present high-resolution quantitative classification and the Mathys results}

Since the Conti spectral type classification relies on the logarithmic ratio of the measured equivalent widths of He I $\lambda 4471$ and $\mathrm{He}$ II $\lambda 4541$, it is essential to investigate the correspondence between the present equivalent width scale and that from similar studies using low-resolution spectra (e.g., Conti \& Alschuler 1971; Conti \& Frost 1977; Mathys 1988, 1989), to provide a useful comparison. We limit ourselves to the study by Mathys (1988), which has the largest overlap with our stellar sample.

In the top panel of Fig. 6, our $E W$ measurements for He I $\lambda 4471$ (asterisks) and He II $\lambda 4541$ (diamonds) are compared to those from Mathys (1988) for nine stars in common. Despite the small number of objects, a clear trend for He II $\lambda 4541$ is visible, indicating that our measurements result in systematically stronger $E W \mathrm{~s}$ than derived by Mathys. A linear fit to the corresponding data confirms this notion, displaying a non-zero offset in the $E W$ scale (in $\AA$ ) for this line given by:

$E W 4541$ (ours) $=(-0.096 \pm 0.06)+(1.01 \pm 0.11)$ EW4541(M88) .

Interestingly, no indication of any shift was found in the $E W$ scale of He I 14471 :

$E W 4471$ (ours) $=(-0.01 \pm 0.06)+(1.01 \pm 0.1) E W 4471(\mathrm{M} 88)$.

These results imply that at least some of our targets might appear as a somewhat earlier spectral type than denoted by Mathys (1988). The data shown in Fig. 6 (lower panel) indicate that our 


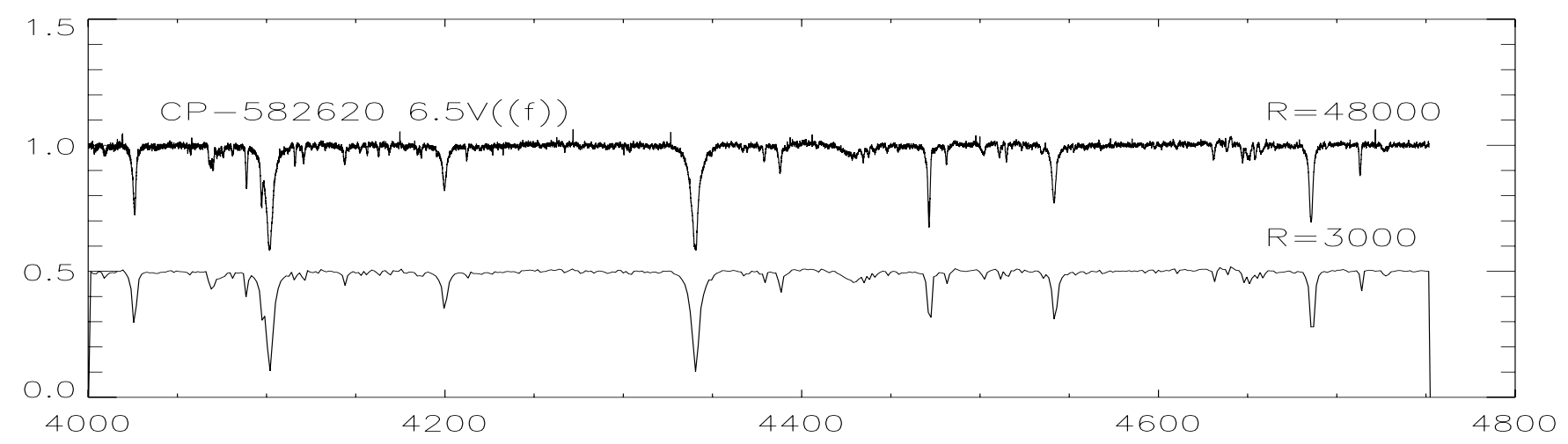

Fig. 5. The spectrum of the narrow-lined star CPD-582620 $\left(v \sin i=60 \mathrm{~km} \mathrm{~s}^{-1}\right)$ at the original resolving power $R=48000$ and degraded to $R=3000$. Spectral classification from Walborn based on low-quality photographic spectra. Note that He I $\lambda 4471$ appears of similar strength as He II $\lambda 4541$ in the degraded spectrum, indicating the O7 subtype.
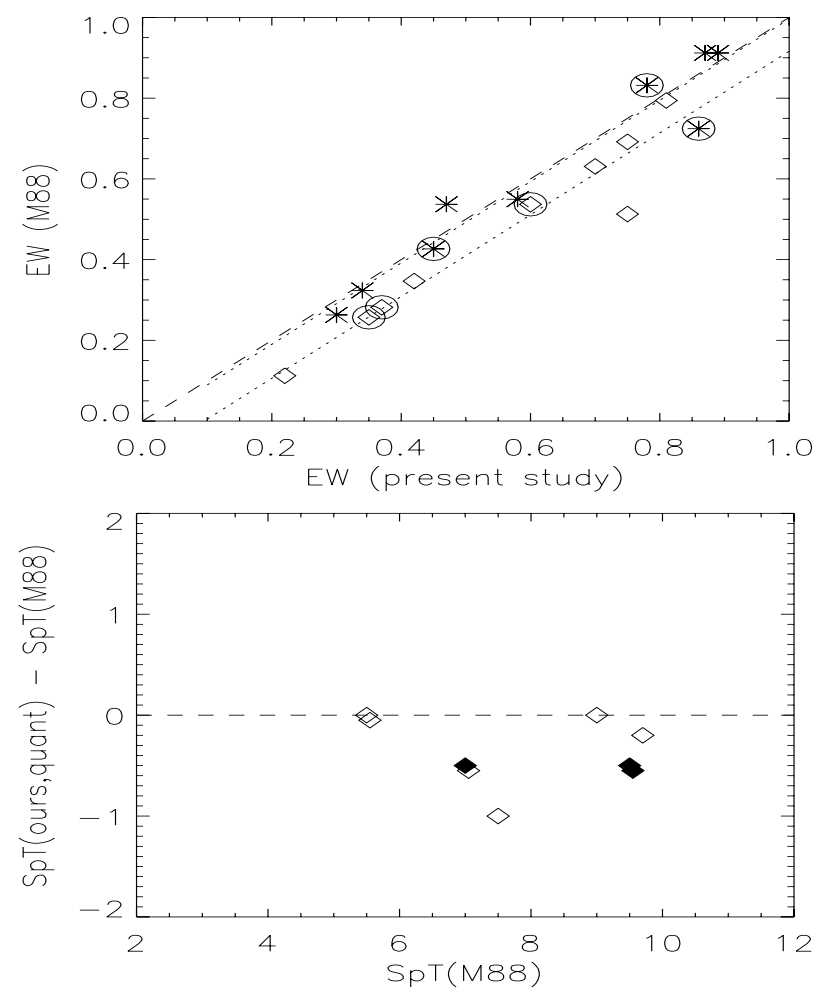

Fig. 6. Top: comparison of our high-resolution equivalent widths (in $\AA$ ) of He I $\lambda 4471$ (asterisks) and He II $\lambda 4541$ (diamonds) with those from Mathys (1988), for nine stars in common. Dotted lines represent linear fits to the corresponding data. Note the non-zero offset in the $E W$ scale of He II $\lambda 4541$. Bottom: comparison of the corresponding highresolution quantitative spectral types. Spectroscopic binaries are highlighted by either large circles (top panel) or filled diamonds (bottom panel).

spectral types tend to be earlier, by half a subtype. Though this discrepancy is comparable to the error budget of our quantitative classification, it might be significant because of its systematic character (see Sect. 6).

Concerning luminosity classes, perfect agreement between the present quantitative luminosity classes and those attributed by Mathys (1988) was established for the nine stars in common.

The discrepancy between our present and the Mathys $E W$ scale for He II $\lambda 4541$ is difficult to interpret. On the one hand, binarity cannot be an issue, because both double and single stars are involved. On the other hand, a higher precision of our measurements can be expected, because of the higher quality of our spectra. This higher quality will allow us to consider the contribution of the broad Stark wings more correctly. Given that in He II $\lambda 4541$ the wings are more extended than in He I $\lambda 4471$ (linear against quadratic Stark effect), we speculate that the effect of a higher $R$ and $S / N$ on the measured $E W$ s will be more pronounced in the former than in the latter line.

To conclude, the luminosity class criterion in the Conti scheme is rather insensitive to changes in $R$, while the spectral type indicator seems to be sensitive, though at a (very) low level.

\section{Correspondence between quantitative and morphological spectral types}

It is important to verify the consisitency of the two alternative schemes for the classification of O-type stars. Earlier studies (Conti \& Leep 1974; Mathys 1988, 1989) have shown that quantitative (either photographic or low-resolution digital) and morphological classification based on photographic data are consistent to within one to two subclasses, with the former being systematically later (on average, by roughly half a spectral subtype).

The same comparison, but between the present quantitative and the present morphological classifications based on our degraded spectra $(R=4000)$, indicates a closer agreement (within \pm 0.5 subtypes) without any systematic trend (Fig. 7, top panel). When considering the correspondence between the high resolution quantitative and morphological spectral types, we find evidence in our data of a systematic difference, where the former is now earlier, by up to 1.5 subtypes $^{8}$ (Fig. 7 , lower panel).

Thus, it appears that because of the higher quality of presentday optical spectra (which increases the accuracy of quantitative measurements and decreases the number of unresolved binary systems), the consistency between the two classification schemes can be significantly improved leading to practically equal results (provided the morphological classification is performed using high-resolution spectra degraded down to $R=4000)$.

8 This finding is a natural consequence of our results from Sect. 4, namely that within the high-resolution spectra He I+II $24026 /$ He I $\lambda 4471$ can appear deeper than He II $\lambda 4200 / \mathrm{He}$ II $\lambda 4541$, although their $E W$ s are smaller. 

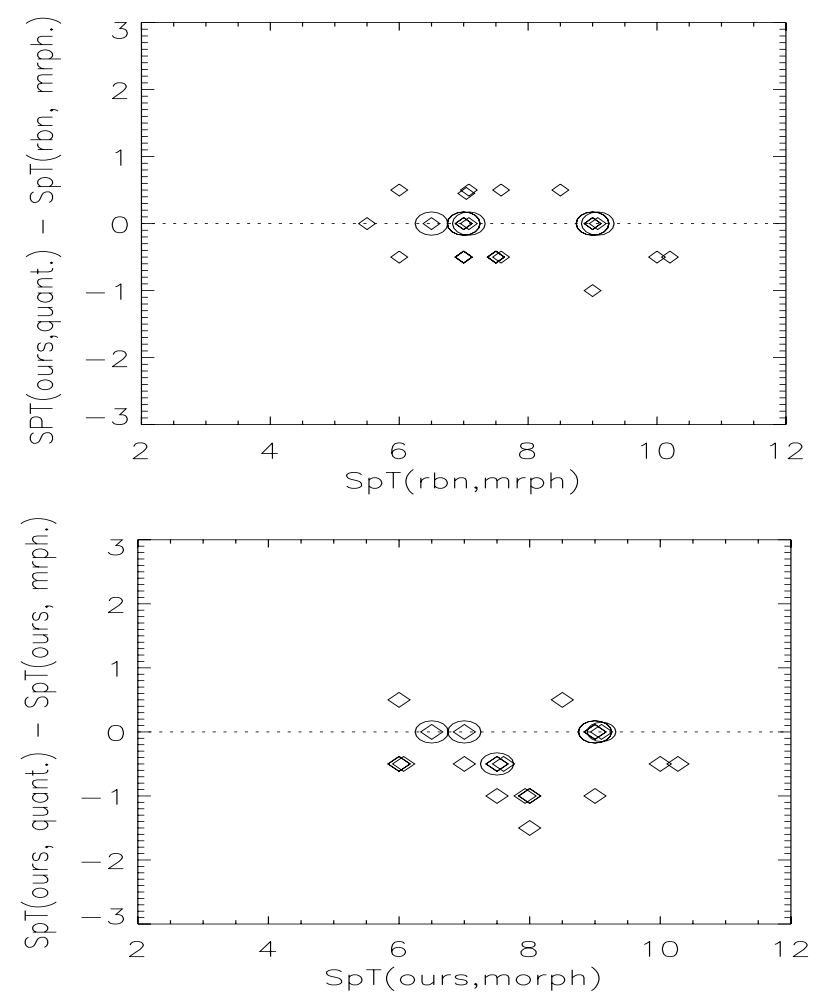

Fig. 7. Top: high-resolution quantitative versus morphological spectral types based on our high-resolution spectra degraded to $R=$ 4000. Bottom: high-resolution quantitative versus high-resolution morphological spectral types.

\section{Summary and future perspectives}

Using high-resolution ESO/MTG FEROS spectra and applying the Walborn and the Conti schemes, we have performed a spectral classification of 19 Galactic O-type dwarfs and giants. We have investigated the correspondence between these highresolution classifications and those attributed by Walborn (1972, 1973) based on low-quality photographic spectra and those assigned by Mathys (1988) using low-resolution digital spectra. In addition, we have also investigated the spectroscopic status of our targets. The main results can be summarised as follows.

1. Spectroscopic binaries. Using photometric/spectroscopic data from the literature in combination with results from a FASTWIND model atmosphere analysis (Paper II), we confirm the binary nature of HD 152723 and HD 152247, provide strong evidence of a companion in HD 93160 and HD 151003, and suspect possible duplicity in HD 69464. Four of these stars are members of cluster/associations and one is a field star. Thus, the binary fraction among our sample is 26 percent if both the cluster members and the field stars are considered. Given that the majority of our targets has never been monitored photometrically or spectroscopically, and that systems with orbital periods too long for an easy detection and too short for a direct angular separation can easily be lost, we consider this estimate as a lower limit. For spectral classification, our analysis shows that binarity may or may not affect the derived spectral type/luminosity class, depending on the magnitude difference, separation, slit orientation etc. In any case, and as might be expected, this effect does not lead to systematic discrepancies (provided these systems are not used as standards), but "only" increases the uncertainty in the derived classification.
2. Correspondence between the present morphological classification and the Walborn results. Within a sample of 14 stars, we found evidence of a systematic discrepancy between our morphological spectral types and those attributed by Walborn, with the former being later by up to 1.5 subtypes (Fig. 1). This discrepancy is larger than the corresponding uncertainties and thus significant. Concerning luminosity classes, no evidence of any systematic difference between our determinations and those from Walborn was found. In our present understanding, the systematic discrepancy in morphological classification is due to an interplay between stellar rotation and spectral resolution (Figs. 2 and 3) as well as technical differences in the classification process of photographic spectrograms and digital spectra (Fig. 4). Another important outcome of our analysis is that at least for narrowlined stars $\left(v \sin i \leq 100 \mathrm{~km} \mathrm{~s}^{-1}\right)$ the "rotational effect" does not necessarily disappear when the high-resolution spectra are degraded to the resolution of the Walborn standards (Figs. 2 and 5).

3. Correspondence between the present quantitative classification and the Mathys results. Within a limited sample of nine stars, we found that the spectral type criterion in the Conti scheme, $\log W^{\prime}$, is sensitive to changes in the spectral resolving power (presumably due to effects on the $E W$ of He II $\lambda 4541$ ), while the luminosity class criterion, $\log W^{\prime \prime}$, is insensitive. In particular, the present high-resolution quantitative spectral types tend to be systematically earlier (by typically half a subtype) than those attributed by Mathys. Although comparable to the corresponding errors, the established discrepancy might be significant because it is systematic.

4. Correspondence between the present quantitative and morphological spectral types. Although different from earlier results, the modern-era quantitative and morphological classifications appear to be internally consistent (provided that the morphological classification is performed using high-resolution spectra degraded to $R=4000$ ). This result, if confirmed by better statistics, might help us to improve the accuracy and consistency of Galactic $\mathrm{O}$ star classification after a corresponding new atlas of standard stars at $R=4000$ has been set up and published (see below).

From the results outlined above, one might conclude that the quantitative classification is more robust than the morphological one. This conclusion, however, would be somewhat premature since it would be based on a very limited sample of stars that furthermore does not include supergiants. On the other hand, a spectral type discrepancy of even half a subtype, if confirmed on the basis of higher quality statistics, might be significant and therefore require the Conti scheme to be updated using the power of high-quality spectral observations and line profile simulations. Such a thorough study is foreseen within our collaboration in the VLT-FLAMES Tarantula Survey (PI: C. J. Evans).

Concerning the morphological classification, a possible solution of the problems identified in the present study requires an update of the corresponding approach. This revision might comprise the following steps: (i) selection and observation (at relatively high spectral resolution and $S / N$ ) of classification standards, which will be used to create a new digital atlas superseding the one of Walborn \& Fitzpatrick (1990); (ii) development of a new classification technique that allows the spectra of both unknown and standard stars to be compared at the same resolution and $v \sin i$; and (iii) reclassification of as many 
Galactic O-stars as possible (observed uniformly and at the same $R$ as the standards) to be used as a firm basis for future studies.

First steps in this direction have been undertaken within the Galactic O-stars Spectroscopic Survey (GOSSS) (PI: J. M. Appelániz) and the VLT-FLAMES Tarantula Survey. Within the GOSSS survey, a digital atlas of Galactic O-type stars from both hemispheres will be created and published, to supersede the one of Walborn \& Fitzpatrick (1990) in terms of both the quality of the data $(S / N \approx 200, R \approx 2500)$ and the number of standard stars included. Within the VLT-FLAMES Tarantula Survey project, on the other hand, we plan to publish a digital atlas of standard O-type stars using high-resolution $(R$ from about 40000 to about 80000$)$, high signal-to-noise $(S / N \approx 500)$ spectra collected from the ESO public archive and the IACOB database (Simón-Díaz et al. 2010). To diminish the combined effects of resolution and rotation and ensure consistency with the modernera quantitative classification, the spectra will be degraded to $R=4000$, and subsequently used to create an atlas of unprecedented quality.

Finally, we point out that although updated with respect to the resolution and rotational effects, the classification of O-type stars might still be subject to significant third parameter effects related to metallicity (see Markova et al. 2009 and references therein).

Acknowledgements. We like to thank our anonymous referee for useful comments and suggestions. N.M. gratefully acknowledges travel grants by the IAC, Tenerife, Spain, and the Catania Observatory, Italy. This investigation was supported by the Bulgarian NSF (contract DO 02-85). S.S.D. and A.H. acknowledge financial support from the Spanish Ministerio de Ciencia e Innovación under the projects AYA2008-06166-C03-01 and the Consolider-Ingenio 2010. Program grant CSD2006-00070: First Science with the GTC (http://www . iac.es/consolider-ingenio-gtc).

\section{References}

Balona, L. A. 1975, MNRAS, 78, 51 Balona, L. A. 1983, MNRAS, 203, 1041

Bouret, J.-C., Lanz, T., \& Hillier, D. J. 2005, A\&A, 438, 301

Conti, P. S., \& Alschuler, W. R. 1971, ApJ, 170, 325

Conti, P. S., \& Ebets, D. 1977, ApJ, 213, 438

Conti, P. S., \& Frost, S. A. 1977, ApJ, 212, 728

Conti, P. S., \& Leep, E. M. 1974, ApJ, 193, 113

Crampton, D. 1971, AJ, 76, 260

Crampton, D. 1972, MNRAS, 158, 85

Duflot, M., Figon, P., \& Mayssonnier, N. 1995, A\&AS, 114, 269

Dufton, P. L., Smartt, S. J., Lee, J. K., et al. 2006, A\&A, 457, 265

Garcia, M., \& Bianchi, L. 2004, AphJ, 606, 497

Garrison, R. F., Hiltner, W. A., \& Schild, R. E. 1977, ApJS, 35, 111
Georgelin, Y. P., \& Georgelin, Y. M. 1970, A\&AS, 3, 1

Gies, D. 1987, ApJSS, 64, 545

Herrero, A., Puls, J., \& Najarro, F. 2002, A\&A, 396, 946

Howarth, I., Siebert, K., Hussain, G., et al. 1997, MNRAS, 284, 265

Feast, W. M. 1958, MNRAS, 118, 618

Feast, W., Stoy, R., Thackeray, A., et al. 1961, MNRAS, 122, 239

Fullerton, A., Gies, D., \& Bolton, C. T. 1996, ApJS, 103475

Kaufer, A., Stahl, O., Tubbesing, S., et al. 1999, The Messenger, 95, 8

Kilkenny, D., van Wyk, F., Roberts, G., et al. 1998, MNRAS, 294, 93

Lefevre, L., Marchenko, S., Moffat, A., et al. 2009, A\&A, 507, 1141

Levato, H., \& Malaroda, S. 1981, PASP, 93, 714

Levato, H., Malaroda, S., Garcia B., et al. 1990, ApJS, 71, 323

Levato, H., Malaroda, S., Morrell, N., et al. 1991, ApJS, 72, 869

Loden, L. O. 1965, ApJ, 141, 668

Marchenko, S., Moffat, A., van der Hucht, K., et al. 1998, A\&A, 331, 1022

Marcolino, W., Bouret, J-C, Martins, F., et al. 2009, A\&A, 498, 837

Markova, N., Bianci, L., Efremova, B., \& Puls, J. 2009, BlgAJ, 12, 21

Martins, F., Schaerer, D., \& Hillier, D.J. 2005a, A\&A, 436, 1049

Martins, F., Schaerer, D., \& Hillier, D.J. 2005b, A\&A, 441, 735

Mason, B. D., Gies, D., Hartkopf, W. I., et al. 1998, ApJ, 115, 821

Mason, B. D., Hartkopf, W. I., Gies, D., et al. 2009 AJ, 137, 3358

Massey, Ph., DeGioia-Eastwood, K., \& Waterhous, E. 2001, ApJ, 121, 1050

Mathys, G. 1988, A\&AS, 76, 427

Mathys, G. 1989, A\&ASS, 81, 237

Moffat, A. F. J. 1977, IBVS.1265, 1

Moffat, A. F. J., \& Seggeviss 1979, A\&A, 70, 69

Morrell, N., Garcia, B., \& Levato, H. 1988, PASP, 100, 143

Morris, P. 1961, MNRAS, 122, 325

Nesterov, V. V., Kuzmin, A. V., Ashimbaeva, N. T., et al. 1995, A\&AS, 110, 367 (III/182)

Penny, L. R. 1996, ApJ, 463, 737

Penny, L. R., \& Gies, D. 2009, ApJ, 700, 844

Penny, L., Gies, D., \& Hartkopf, W. 1993, PASP, 104, 588

Penny, L., Sprague, A., Seago, G. et al. 2004, ApJ 617, 1316

Peton-Jonas, D. 1981, A\&AS, 45, 193

Puls, J., Kudritzki, R.-P., Herrero, A., et al. 1996, A\&A, 305, 171

Puls, J., Urbaneja, M., Venero, R., et al. 2005, A\&A, 435, 669

Puls, J., Vink, J., \& Najarro, F. 2008, A\&ARv, 16, 209

Rabaud, D. 1996, A\&A, 315, 384

Reed, B. C. 2000 , AJ, 119,1855

Reed, B. C. 2005, AJ, 130, 1652

Repolust, T., Puls, J., \& Herrero, A. 2004, A\&A, 415, 349

Sana, H., Gosset, E., Naze, Y., et al. 2008, MNRAS, 386, 447

Schild, R.,Hiltner, W., \& Sanduleak, N. 1969, ApJ, 156, 609

Simón-Díaz, S., Castro, N., Garcia, M., et al. 2010 [arXiv: 1009. 5824]

Solivella, G. R., \& Niemela, V. S. 1986, RMxAA, 12, 188

Turner, D.G. 1977, AJ, 82, 163

Turner, N., Ten Brummelaar, T., Roberts, L., et al. 2008, AJ, 136. 554

Uesugi, A., \& Fukuda, I. 1995, Revised Catalogue of Rotational Velocities (III/63B)

Walborn, N. R. 1971, ApJS, 23, 257

Walborn, N. R. 1972, AJ, 77, 312

Walborn, N. R. 1973, AJ, 78, 1067

Walborn, N. R., \& Fitzpatrick, E. 1990, PASP, 102, 379

Walborn, N., Howarth, I., Lennon, D., et al. 2002, AJ, 123, 2754

Walborn, N., Sota, A., Apellaniz, J. M., et al. 2010, ApJ, 711, L143

Pages 10 to 15 are available in the electronic edition of the journal at http://www . aanda. org 


\section{Appendix A: Comments on individual stars}

Table A.1. Equivalent widths (in $\AA$ ) and equivalent width ratios as measured for the O-type stars in our sample.

\begin{tabular}{lcccccccc}
\hline \hline Star & He I $\lambda 4471$ & He II $\lambda 4541$ & He I $\lambda 4143$ & Si IV $\lambda 4089$ & $\log E W(4686)^{a}$ & $\log W^{\prime} b$ & $\log W^{\prime \prime} c$ & Quant. class. \\
\hline HD 64568 & - & 0.84 & - & - & -0.46 & -38 & O5.5 V \\
HD 93204 & 0.34 & 0.81 & - & - & -0.17 & -0.38 & O5.5 III \\
HD 93843 & 0.30 & 0.70 & - & - & -0.72 & -0.37 & O6.5 V/III \\
CPD -592600 & 0.43 & 0.63 & - & - & -0.24 & -0.17 & O6.5 V \\
HD 63005 & 0.59 & 0.80 & - & - & -0.12 & -0.13 & O6.5 III \\
HD 152723 & 0.45 & 0.60 & - & - & -0.36 & -0.12 & O7 V \\
HD 93160 & 0.55 & 0.62 & 0.15 & 0.17 & & -0.05 & 0.05 & O. \\
HD 94963 & 0.59 & 0.61 & 0.11 & 0.23 & & -0.01 & 0.32 & O7-7.5 I/III \\
CPD -58 2620 & 0.64 & 0.76 & 0.12 & 0.22 & & -0.07 & 0.26 & O7 III \\
HD 69464 & 0.64 & 0.66 & 0.10 & 0.19 & & -0.01 & 0.28 & O7-7.5 III \\
HD 93222 & 0.60 & 0.65 & 0.12 & 0.23 & & -0.03 & 0.28 & O7 III \\
HD 91824 & 0.58 & 0.75 & 0.13 & 0.15 & -0.05 & -0.11 & O6.5 V \\
CD -43 4690 & 0.47 & 0.75 & 0.08 & 0.16 & -0.23 & -0.20 & O6.5 V/III \\
HD 92504 & 0.87 & 0.42 & 0.29 & 0.37 & & 0.32 & 0.11 & O9 III/V \\
HD 151003 & 0.78 & 0.37 & 0.25 & 0.44 & & 0.32 & 0.24 & O9 III \\
HD 152247 & 0.86 & 0.35 & 0.30 & 0.47 & & 0.39 & 0.19 & O9 III \\
HD 302505 & 0.79 & 0.55 & 0.22 & 0.37 & & 0.16 & 0.22 & O8 III \\
CPD -44 4865 & 0.89 & 0.22 & 0.32 & 0.50 & & 0.61 & 0.19 & O9.5 III \\
HD 69106 & 0.95 & 0.17 & & & & 0.74 & O9.7 \\
\hline
\end{tabular}

Notes. Accuracy of individual equivalent width measurements: 0.02 to $0.07 \mathrm{dex}$, depending on the strength of the line (the stronger the line, the higher the accuracy). Quantitative classification accounting for the error in $\log W^{\prime}(\sim \pm 0.03$ dex $)$ and $\log W^{\prime \prime}(\leq \pm 0.05$ dex $)$. ${ }^{(a)} \log E W(\lambda 4686)$ determines the luminosity class for stars of spectral types O6.5 and earlier (see Mathys 1988). ${ }^{(b)} \log W^{\prime}=\log E W(\mathrm{HeI} \lambda 4471)-\log E W(\mathrm{HeII} \lambda 4541)$ determines the spectral type (see Conti \& Alschuler 1971 and Mathys 1988). ${ }^{(c)} \log \mathrm{W}^{\prime \prime}=\log E W(\mathrm{HeI} \lambda 4143)-\log E W$ (SiIV $\left.\lambda 4089\right)$ determines the luminosity class for stars of O7 and later (Conti \& Alschuler 1971).

HD 64568 - This star was classified as O4 V((f)) (Garrison et al. 1977), O5 V (Loden 1965; Crampton 1972; Peton-Jonas 1981), and O3 V((f*)) (Walborn et al. 2002). The FUV/UV spectrum is also consistent with the $\mathrm{O} 3 \mathrm{~V}((\mathrm{f} *))$ classification (Garcia \& Bianchi 2004). The visual appearance of our spectrum is identical to that shown in Walborn et al., suggesting the same $\mathrm{O} 3 \mathrm{~V}\left(\left(\mathrm{f}^{*}\right)\right)$ designation. The discrepant spectral types assigned at different observational epochs might indicate that HD 64568 is a binary. This possibility seems to be supported by the detected $V_{\mathrm{r}}$ - variability (Solivella \& Niemela 1986), but the good agreement between the model and the observed spectra (Paper II) argues in favour of the single-star hypothesis. The star has not been photometrically monitored and its short/long-term behaviour is completely unknown. Lacking direct evidence of possible binarity, we are inclined to consider HD 64568 as a single object with $V_{\mathrm{r}}$ - variations caused more likely by stellar pulsations (and/or wind variability). In this case then, the spectral type discrepancy could be due to reasons other than spectroscopic binarity.

HD 93204 is classified as O5 V((f)) by Walborn (1973) and considered as a standard of this subtype. Walborn et al. (2002) repeated this designation, whereas Mathys (1988) proposed O5.5 V((f)). Our high-resolution spectrum is identical to the one shown in Walborn et al. (2002). Consequently, we should denote the star O5 V((f)), but in our spectra, as well as in Walborn's, $\mathrm{He}$ I+II $\lambda 4026$ is of similar strength as He II $\lambda 4200$, which is the definition of an O6 subtype. The measured $\log W^{\prime}=-0.38$ and $\log E W(4686)=-0.17$ imply the $05.5 \mathrm{~V}$ classification. Within the limits of their $V$-band speckle interferometric observations, Mason et al. (2009) conclude that HD 93204 is a visually single object. To our knowledge, this star has never been monitored photometrically or spectroscopically. In Paper II, we have no problems obtaining good fits to all strategic helium and hydrogen lines using one set of parameters (including $V_{\mathrm{r}}$ and $v \sin i$ ). Projected rotational velocities measured at different observational epochs agree perfectly (e.g., $v \sin i=130$, Conti \& Ebbets 1977; $v \sin i=130 \mathrm{~km} \mathrm{~s}^{-1}$, Penny 1996). All this suggests that HD 93204 is a spectroscopically single object.

We note that Walborn et al. (2010) have recently reclassified HD 93204 from O5((f)) to O5.5((fc)), where "fc" indicates the presence of C III $\lambda \lambda 4647-4650-4652$ emission lines of comparable strength to those of N III $\lambda \lambda 4634-4640-4642$. Interestingly, in our high-resolution spectrum $(R$ about ten times higher than that in the Walborn et al. observations), no sign of any C III emission can be seen, suggesting that the amount of C III emission is temporally variable in HD 93204. Similar results have been reported for three stars of the Of?n category: HD 108, HD 191612 , and CPD -282561 (Walborn et al. 2010). The physical reason for the variable C III emission is still not well understood but given that HD 108 and HD 191612 have been recognized as long period binaries with magnetic fields, an interpretation in terms of orbital phase occultation of a localized C III emitting region has been suggested (Walborn et al. 2010 and references therein). If confirmed by means of a more detailed investigation, the single nature of HD 93204 would require an explanation of the variable C III emission that is different from that suggested by Walborn et al. (2010).

HD 93843 - This star was initially classified as O6 III(f) (Walborn 1972), and later on reclassified as O5 III(f) (Walborn 1973). Applying the quantitative approach, Mathys (1988) assigned O5.5 III(f). The morphology of our spectrum agrees well with the O6 III(f) designation but with C III in emission. Following the definition of the new Ofc category (see above), we would denote it by O6 III(fc), that is one subtype later than the designation by Walborn et al. (2010), which is O5 III(fc). The measured $\log W^{\prime}=-0.37$ is consistent with the O5.5 subtype. In the interferometric survey of Mason et al. (2009), 
HD 93843 is flagged as an object with a "null companion detection". The star does not seem to show variations in its $V_{\mathrm{r}}$ (Gies 1987). The model atmosphere analysis does not reveal any discrepancy in the shifts and widths of strategic lines (Paper II). Individual $v \sin i$ - estimates derived in different observational epochs agree within the corresponding uncertainties (e.g., $v \sin i=120 \mathrm{~km} \mathrm{~s}^{-1}$, Conti \& Ebbets 1977; $v \sin i=95 \mathrm{~km} \mathrm{~s}^{-1}$, Howarth et al. 1997; $v \sin i=100 \mathrm{~km} \mathrm{~s}^{-1}$, Penny 1996). We suggest that HD 93843 is a spectroscopically single object.

CPD -592600 - Walborn (1973) classified this star as O6 V((f)). The morphology of our spectrum resembles that of HD 101190, the primary standard for the O6 V((f)) subtype (Walborn \& Fitzpatrick 1990), and consequently we assign O6 $\mathrm{V}((\mathrm{f}))$ to our target as well; $\log W^{\prime}=-0.17$ and $\log E W(4686)=-0.24$ indicate O6.5 V/III. A literature research shows that CPD -592600 has never been observed systematically. A comparison of individual $v \sin i$-estimates does not show any significant variations in this parameter (e.g., $v \sin i=140 \mathrm{~km} \mathrm{~s}^{-1}$, Conti \& Ebbets 1977; $v \sin i=142 \mathrm{~km} \mathrm{~s}^{-1}$, Howarth et al. 1997; $v \sin i=135 \mathrm{~km} \mathrm{~s}^{-1}$, Penny \& Gies 2009). This finding together with the good agreement between synthetic and observed strategic lines (Paper II) suggests that CPD 592600 is likely a single object.

HD 63005 was classified as O7 by Loden (1965) and O6 V((f)) by Garrison et al. (1977). The general appearance of our spectrum agrees well with the O7.5 designation: He I $\lambda 4471$ is slightly deeper than He II $\lambda 4541$, and there are both weak N III lines in absorption and missing C III $\lambda 4650$ absorption. Since He II $\lambda 4686$ is comparable in strength to He II $\lambda 4541$, the star might be a dwarf, but the strength of the N III $\lambda \lambda 4634-$ 4640-4642 emission lines are more consistent with a luminosity class "III" rather than "V" designation. Based on these considerations, we adopt $\mathrm{O} 7.5 \mathrm{~V}((\mathrm{f}))$, but draw attention to its somewhat strong N III emission. The measured $\log W^{\prime}=-0.13$ and $\log E W(4686)=-0.12$ indicate O6.5 V. No reports about duplicity were found in the literature. The model atmosphere analysis has not revealed any spectral discrepancy (Paper II). Projected rotational velocities estimated at different epochs agree perfectly (e.g., $v \sin i=73 \mathrm{~km} \mathrm{~s}^{-1}$, Penny 1996; $v \sin i=74 \mathrm{~km} \mathrm{~s}^{-1}$, Howarth et al. 1997; $v \sin i=80 \mathrm{~km} \mathrm{~s}^{-1}$, Penny 2004). With all this in mind, we consider HD 63005 a spectroscopically single object.

HD 152723, SB1 - This star was classified as O7 by Schild et al. (1969) and O6.5 III(f) by Walborn (1972) and Garrison et al. (1977). The appearance of our spectrum is consistent with the O6.5 III(f) designation: He I+II $\lambda 4026$ is slightly deeper than He II $\lambda 4200$; He I $\lambda 4471$ and He II $\lambda 4686$ are weaker than He II $\lambda 4541$, and there is weak N III emission. The classification determined by means of the measured $\log W^{\prime}=-0.12$ and $\log E W(4686)=-0.36$ is also O6.5 III. Mason et al. (2009) resolved HD 152723 as a visual binary with an angular separation of 0.098 ". Based on observed $V_{\mathrm{r}}$ - variability, Fullerton et al. (1996) suggested that HD 152723 is a SB1. This view was supported by Lefevre et al. (2009) who discovered photometric variations with a period of $0.395^{d}$. The discrepant strengths and widths of strategic helium and hydrogen lines (Paper II) also argue in favour of the binary hypothesis. Individual $v \sin i$ - estimates available in the literature however do not deviate significantly, e.g., $v \sin i=110 \mathrm{~km} \mathrm{~s}^{-1}$, Balona (1975); $v \sin i=130 \mathrm{~km} \mathrm{~s}^{-1}$, Conti \& Ebbets (1977); $v \sin i=111 \mathrm{~km} \mathrm{~s}^{-1}$, Howarth et al. (1997); $v \sin i=123 \mathrm{~km} \mathrm{~s}^{-1}$, Penny (1996). We consider HD 152723 as a single-lined SB.

HD 93160, SB1 - Walborn (1972) denoted this star by O6 III(f). From the visual inspection of our spectrum, we classify it as $\mathrm{O} 7 \mathrm{III}(\mathrm{f})$ : He I $\lambda 4471$ of similar strength as He II $\lambda 4541$; weak He II $\lambda 4686$ absorption in combination with weak N III $\lambda \lambda 4634-4640-4642$ emission. The quantitative diagnostics, $\log W^{\prime}=-0.05$ and $\log W^{\prime \prime}=0.05$, indicate a less luminous object, namely a dwarf, of the same 07 subtype. The spectral type discrepancy between the present morphological and Walborn's classification might indicate that HD 93160 is a spectroscopic binary. This possibility was initially suggested by Gies (1987) based on $V_{\mathrm{r}}$ - arguments, the latter however being questioned by Levato et al (1991). Our model atmosphere analysis has revealed a number of discrepancies in the optical spectrum, which might be interpreted as an indication of a close companion (Paper II). The binary hypothesis is supported by the large differences in $v \sin i$ derived in different observational epochs: e.g., $v \sin i=180 \mathrm{~km} \mathrm{~s}^{-1}$, (Conti \& Ebbets 1977); $v \sin i=205 \mathrm{~km} \mathrm{~s}^{-1}$, (Uesugi \& Fukuda 1995); $v \sin i=145 \mathrm{~km} \mathrm{~s}^{-1}$ (Paper II). This star is very likely a SB1.

HD 94963 was previously classified as O6.5 III(f) by Walborn (1973) and O7 III by Garrison et al. (1977). Our spectrum of this star agrees with the O7.5 II subtype. Within the corresponding error, $\log W^{\prime}=-0.01$ is consistent with the O7-7.5 subtype, while $\log W^{\prime \prime}=0.32$ corresponds to a supergiant or at least a giant. The discrepant classification attributed by various authors at different epochs might indicate that HD 94963 is a spectroscopic binary. This possibility seems to be supported by the large spread in individual $v \sin i$ - estimates available in the literature (e.g., $v \sin i=130 \mathrm{~km} \mathrm{~s}^{-1}$, Uesugi \& Fukuda 1995; $v \sin i=90 \mathrm{~km} \mathrm{~s}^{-1}$, Conti \& Ebbets 1977). However, the star is regarded as photometrically stable (Kilkenny et al. 1998), with a constant $V_{\mathrm{r}}$ (Gies 1987). In addition, the model atmosphere analysis does not reveal any discrepancy except for one: the He II $\lambda 4686$ absorption line is peculiarly weak and narrow with a core that is red-shifted with respect to the position of the other spectral lines (Paper II). If not caused by wind asymmetries, the latter result might indicate a composite profile, consisting of an emission feature superimposed on the blue wing of an absorption profile. At least at present, we consider HD 94963 a single object with an asymmetric wind.

CPD -582620 was classified as O6.5 V((f)) by Walborn (1973) and O8 III by Morrell, Garcia, Levato (1988). The morphology of our spectrum is similar to that of $\lambda$ Ori, the classification standard for O8 III((f)) (Walborn \& Fitzpatrick 1990). However, in $\lambda$ Ori as well as in CPD -582620 the N III $\lambda \lambda 4634-4640$ 4642 emission lines are very weak, and He II $\lambda 4686$ is deeper than He II $\lambda 4541$, suggesting the "V((f))" rather than "III(f)" designation. Thus, we classify this star as $08 \mathrm{~V}((\mathrm{f}))$. From the measured equivalent widths, we estimate $\log W^{\prime}=-0.07$ and $\log W^{\prime \prime}=0.26$, which is indicative of O7 III. CPD -582620 is a member of a visual binary with 3.7 arcsec separation. The star has been recognised "as a possible radial-velocity variable", which however "does not show any obvious orbital trend in velocity from night to night" (Penny et al. 1993). No information about systematic photometric variations was found in the 
literature but a comparison of several $U B V$ estimates listed in the Reed (2005) catalogue (V/125) suggests that this star might be photometrically variable. The excellent agreement between theoretical and observed strategic lines suggests that it is more likely to be a single object. Lacking any evidence of binarity, we consider CPD -582620 as a spectroscopically single object, but draw attention to the discrepancies in luminosity class between the various spectral diagnostics.

HD 69464, SB2? - Walborn (1972) classified this star as O6.5 $\mathrm{Ib}(\mathrm{f})$, and defined it as a classification standard for this spectral type/luminosity class (Walborn \& Fitzpatrick 1990). Garrison et al. (1977) attributed O7 III(f). The visual appearance of our spectrum is similar to that of the spectrum shown in Walborn \& Fitzpatrick (1990), with one important difference: in the latter, He II $\lambda 4686$ is completely filled in by wind emission, while in our spectrum it appears as a weak absorption feature. Based on this notion and accounting for He I $\lambda 4471$ being somewhat deeper than He II $\lambda 4541$ in our spectrum as well as in that of Walborn \& Fitzpatrick (1990), we reclassify the star from O6.5 Ib(f) to O7.5 II(f). Within the corresponding error, $\log W^{\prime}=-0.01$ indicates the O7-7.5 subtype. In contrast, $\log W^{\prime \prime}=0.28$ is consistent with the star being a giant. Spectral anomalies such as those outlined above are usually interpreted as an indication of a companion. Unfortunately, HD 69464 has never been observed systematically, and no direct evidence in support or against this possibility exists. Individual $v \sin i$ determinations (e.g., $v \sin i=71 \mathrm{~km} \mathrm{~s}^{-1}$, Howarth et al. 1997; $v \sin i=82 \mathrm{~km} \mathrm{~s}^{-1}$, Penny 1996) and $V_{\mathrm{r}}$ - estimates (e.g., $V_{\mathrm{r}}=43 \mathrm{~km} \mathrm{~s}^{-1}$, Crampton 1972; $V_{\mathrm{r}}=45 \mathrm{~km} \mathrm{~s}^{-1}$, Paper II) seem to agree well, but this may still be due to observational selection. The correspondence between synthetic and observed lines is also good, except for He II 24686 : the observed feature appears peculiarly narrow and weak and may show an inverse P Cygni profile (Paper II). If not due to wind variability or asymmetries, the latter finding would suggest that HD 69464 is a SB2. Systematic spectral/photometric observations are needed to check this possibility in the future.

HD 93222 - Classified as O7 III((f)) by Walborn (1971, 1972), and again by Levato \& Malaroda (1981) and Walborn \& Fitzpatrick (1990). However, Massey et al. (2001) attributed O8 III((f)). The appearance of our spectrum differs significantly from that shown in Walborn \& Fitzpatrick (1990), and resembles much more the spectral morphology of the O8 III((f)) standard $\lambda$ Ori, which we adopt in our classification. The measured $\log W^{\prime}=-0.03$ and $\log W^{\prime \prime}=0.28$ indicate a slightly earlier subtype, namely O7 III. HD 93222 is considered as non-variable in both photometry (e.g., Moffat 1977; Moffat \& Seggeviss 1978 ) and $V_{\mathrm{r}}$ (e.g., Levato et al 1990). $v \sin i$ - estimates from different observational epochs agree quite well (e.g., $v \sin i=65$, Conti \& Ebbets 1977; $v \sin i=77 \mathrm{~km} \mathrm{~s}^{-1}$, Howarth et al. 1997; $v \sin i=77 \mathrm{~km} \mathrm{~s}^{-1}$, Penny 1996). The model atmosphere analysis does not uncover any spectral discrepancy (Paper II). All this suggests that HD 93222 is a spectroscopically single star.

HD 91824 - Walborn (1972) denoted this star by O7 V((f)), confirmed by Garrison et al. (1977). From a visual inspection of our spectrum, we suggest O8 V((f)) since He II $\lambda 4686$ and $\mathrm{He}$ I $\lambda 4471$ are deeper than He II $\lambda 4541$; numerous $\mathrm{N}$ III lines are in absorption, and weak C III in absorption and weak N III $\lambda \lambda 4634-4640-4642$ are in emission. The measured $\log W^{\prime}=-0.11$ and $\log E W(4686)=-0.05$ indicate 06.5 V. HD 91824 was found to be an irregular variable in both photometry (Lefevre et al. 2009) and $V_{\mathrm{r}}$ (Feast 1958). Since the model atmosphere analysis (Paper II) as well as the classification analysis (present study) did not reveal any spectral discrepancy, we suggest that this variability is more likely due to stellar pulsation than to binarity. This possibility is further supported by the good agreement between individual $v \sin i$ - estimates: $v \sin i=67 \mathrm{~km} \mathrm{~s}^{-1}$ (Penny 1996) and $v \sin i=65 \mathrm{~km} \mathrm{~s}^{-1}$ (Howarth et al. 1997).

CD -434690 - Classified as O7.5 III(f) by Crampton (1971) and thereafter by Mathys (1988). The morphology of our spectrum agrees well with the 07 III(f) designation: He I $\lambda 4471$ is as deep as He II 24541 ; N III is in emission, and He II $\lambda 4686$ is somewhat weaker than He II $\lambda 4541$. We measure $\log W^{\prime}=-0.20$ and $\log E W(4686)=-0.23$, which is indicative of the O6.5 V/III type. This star has never been observed systematically, neither photometrically nor spectroscopically. A comparison of individual data provided in the Reed (2005) catalogue did not reveal any significant variations in $V$ and $B-V$ on a longer (year) timescale. Based on several snapshots observations, Crampton (1972) conclude that this star is not a $V_{\mathrm{r}}$ - variable. The model atmosphere analysis does not reveal any spectral discrepancy (Paper II). We consider CD -434690 as a spectroscopically single object.

HD 92504 was denoted by $08.5 \mathrm{~V}$ (Walborn 1973), and this classification was repeated by Garrison et al. (1977). Turner (1977) assigned O9 V. The visual appearance of our spectrum is identical to that of the O8.5 V standard HD 46149 (Walborn \& Fitzpatrick 1990). We measure $\log W^{\prime}=0.32$ and $\log W^{\prime \prime}=0.11$, indicative of the $\mathrm{O} 9 \mathrm{III} / \mathrm{V}$ type. Individual $U B V$ measurements listed in the Reed (2005) catalogue suggest that the star is photometrically stable on a long (year) timescale. Individual $V_{\mathrm{r}}$ estimates given in various catalogues (e.g., III/190 - Duflot et al. 1995; V/125 - Reed 2005) agree well. The model atmosphere analysis has not revealed any discrepancy in the spectrum (Paper II). This star is likely a single object.

HD 151003, SB1? was classified as O9 II (Walborn 1973), O9.5 III (Mathys 1988) and O9 Ib (Garrison et al. 1977). The morphology of our spectrum is in-between that of the classification standards HD 148546 (O9 Ia) and $\iota$ Ori (O9 III) (Walborn \& Fitzpatrick 1990), suggesting an intermediate O9 II classification. $\log W^{\prime}=0.32$ and $\log W^{\prime \prime}=0.24$ indicate O9 III. The differences in the spectral classifications of HD 151003 might indicate that it has a composite spectrum caused by binarity. Within the limits of the Mason et al. $V$-band interferometry, no indication of any companion was found. In the Turner et al. (2008) adaptive optics $I$-band photometric survey the star is flagged with "V", i.e., "one or more additional spectroscopic companions identified". Based on $V_{\mathrm{r}}$ - arguments, Gies (1987) suspected that HD 151003 is a SB1. This view is supported by discrepant widths of strategic helium and Balmer lines (Paper II). Thus, it seems likely that HD 151003 is an SB1. Systematic photometric and/or radial velocity observations are needed to convincingly prove this possibility.

HD 152247, SB2 - According to Walborn (1973), this star is a O9.5II/III and, similarly, a O9.5 III following 
Mathys (1988). Our spectrum is almost identical to that of HD 151003, suggesting the same O9 II classification. The measured $\log W^{\prime}=0.39$ is consistent with the O9 subtype; $\log W^{\prime \prime}=0.19$ corresponds to the luminosity class "III" rather than "II". Balona (1983) reported that the visual brightness of HD 152247 is stable to within \pm 0.05 mag. The star has never been monitored spectroscopically, but a comparison of individual estimates from the literature shows that its $v \sin i$ appears to be stable (e.g., $v \sin i=110 \mathrm{~km} \mathrm{~s}^{-1}$ Conti \& Ebbets 1977; $v \sin i=120 \mathrm{~km} \mathrm{~s}^{-1}$ Penny 1996; $v \sin i=112 \mathrm{~km} \mathrm{~s}^{-1}$ Howarth et al. 1997), while its $V_{\mathrm{r}}$ varies (Rabaud 1996; Sana et a. 2008). On the basis of the detection of an additional weak absorption component in He II 14686, Sana et a. (2008) suggested that HD 152247 is a SB2 consisting of an O9 III and an O9.7 V component. This result is additionally supported by detected discrepancies in the widths of helium and Balmer lines (Paper II). HD 152427 is definitely a SB2, where the secondary is significantly fainter (by 3 or more magnitudes to be consistent with the "null companion detection" of Mason et al. 2009) than the primary.

HD 302505 - Classified as O9.5 III by Georgelin \& Georgelin (1970) and O8.5 III by Garrison et al. (1977). Crampton (1972) listed it as B2 without, however, any reference to the original source. Our spectrum, visually, resembles that of $\iota$ Ori, the classification standard of the O9 III type (Walborn \& Fitzpatrick 1990). $\log W^{\prime}=0.16$ and $\log W^{\prime \prime}=0.22$ indicate O8 III. The lack of consistency between the assigned spectral types might be interpreted as evidence of a companion. However, this view is not supported by the close agreement between the model and the observed spectra (Paper II). Individual $U B V$ measurements listed in the Reed (2005) catalogue suggest that HD 302505 is photometrically stable on a longer timescale (within $\Delta V \leq 0.1 \mathrm{mag}$, $\Delta(B-V) \leq 0.1 \mathrm{mag})$. Thus, we consider this star a single object, but future photometric and radial-velocity monitoring is required to check this view.

CPD -44 4865 has been classified as 09.5 Ib by Feast et al. (1961); Mathys (1988) reclassified it as O9.7 III. The general appearance of our spectrum resembles that of HD 48434, the classification standard for B0 III (Walborn \& Fitzpatrick 1990). The only noticeable difference is that in CPD -444865 , the lines He II $\lambda 4200$ and He II $\lambda 4541$ are definitely present, while they are missing in HD 48434. $\log W^{\prime}=0.61$ and $\log W^{\prime \prime}=$ 0.19 indicate O9.5 III, in good agreement with the classification by Mathys (1988). To our knowledge, CPD -444865 has never been systematically observed. In the Reed catalogue (2005), it is flagged as "RV var", but no reference to the corresponding source(s) is provided. A comparison of individual $V_{\mathrm{r}}$ - estimates from different observational epochs does not show any significant variations (e.g., $V_{\mathrm{r}}=40 \mathrm{~km} \mathrm{~s}^{-1}$, the GCMRV; $V_{\mathrm{r}}=39.3 \mathrm{~km} \mathrm{~s}^{-1}$, the Reed 2005 catalogue). The model atmosphere analysis has not posed any problem: excellent fits were obtained for all strategic lines with one set of parameters (Paper II). All this suggests that CPD -44 4865 is likely a spectroscopically single object.

HD 69106 - Morris (1961) classified this star as B0.5 III; Garrison et al. (1977) reclassified it as B0.5 IVnn. Our spectrum resembles that of $\tau$ Sco, the classification standard for B0.2 V (Walborn \& Fitzpatrick 1990). The measured $\log W^{\prime}=0.74$ corresponds to O9.7. A literature research shows that HD 69106 has been recognised as a photometrically variable star, with a period of $1.48 \mathrm{~d}$ (Marchenko et al 1998). The star has not been monitored spectroscopically. Individual $v \sin i$ - estimates agree well (e.g., $v \sin i=329 \mathrm{~km} \mathrm{~s}^{-1}$, Howarth et al. 1997; $v \sin i=316 \mathrm{~km} \mathrm{~s}^{-1}$, Balona 1975; $v \sin i=328 \mathrm{~km} \mathrm{~s}^{-1}$, Conti $\&$ Ebbets 1977). Excellent fits have been derived for all strategic lines in the spectrum (Paper II). In the absence of direct evidence of binarity, we suggest that HD 69106 is more likely a single very late O-type dwarf undergoing stellar pulsations. 


\section{Appendix B: High-resolution atlas of our sample of stars}

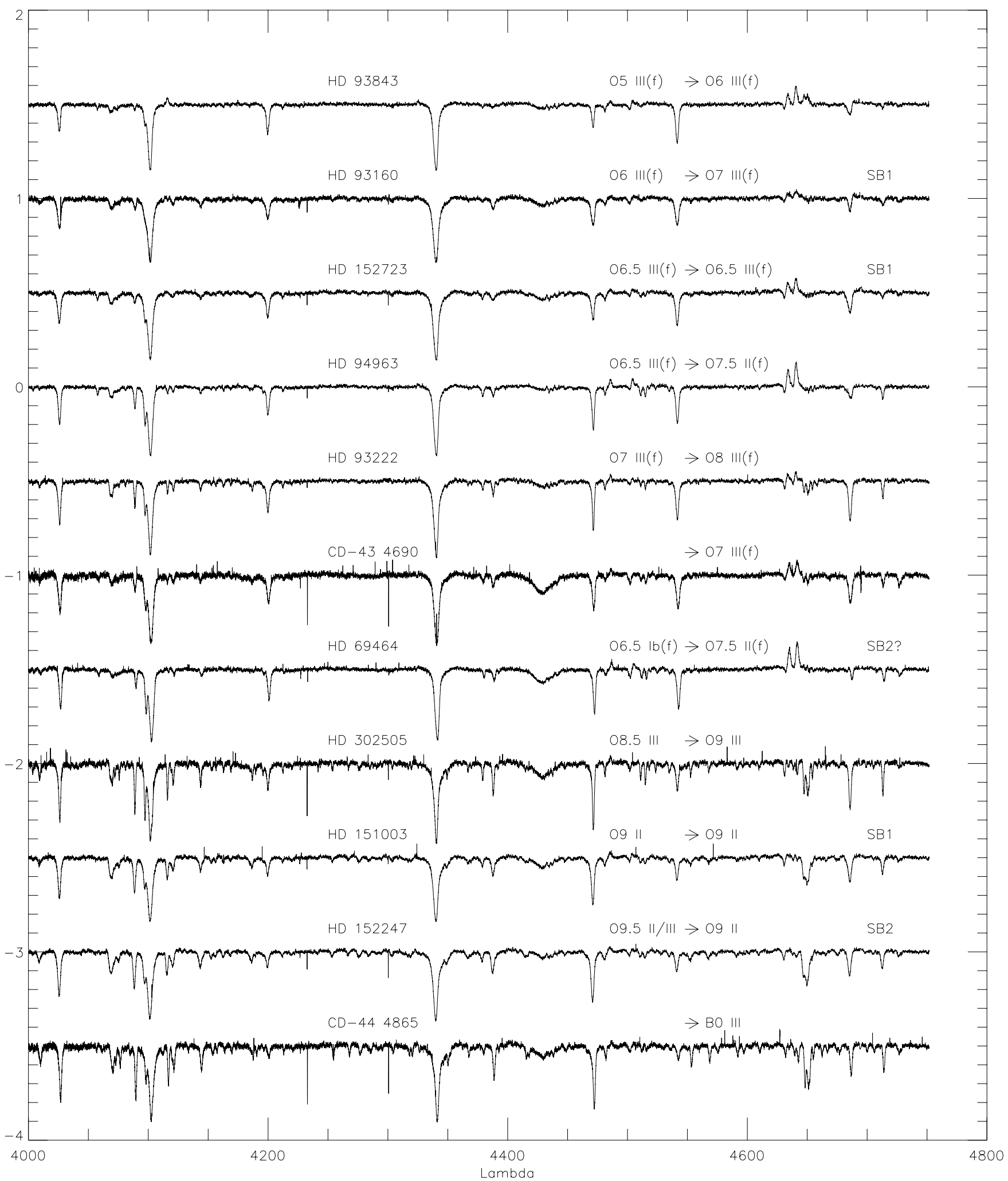

Fig. B.1. High-resolution spectra of sample stars which have been classified originally as giants. Morphological classification as provided by Walborn $(1972,1973)$ using low resolution photographic spectra (with individual data from Garrison et al. 1977), together with our morphological classification based on high-resolution spectra. Confirmed/suspected SB1 and SB2 are also indicated. 
N. Markova et al.: Spectral classification of Galactic O-stars

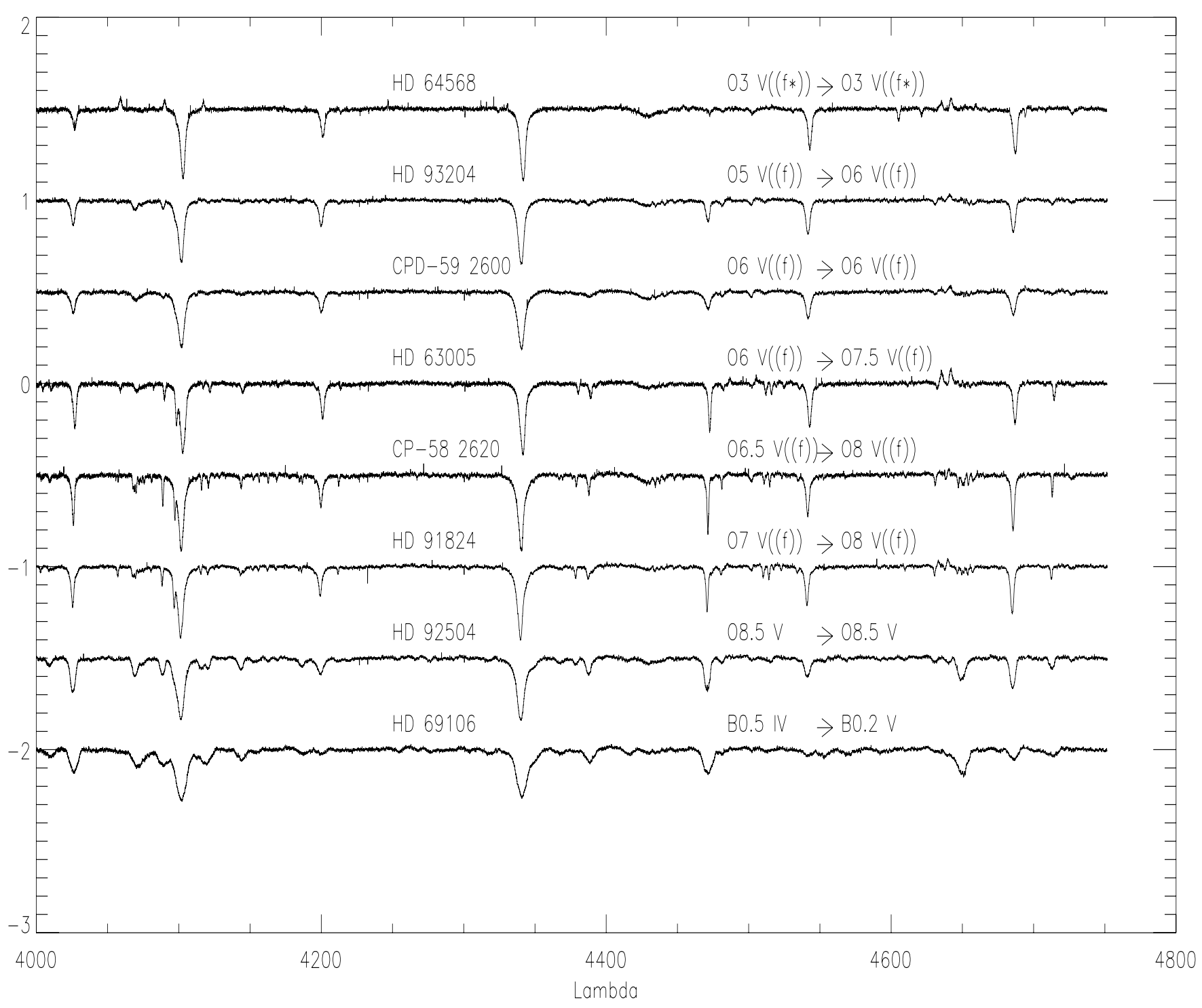

Fig. B.2. As Fig. B.1, but for stars originally classified as dwarfs. 